ARTICLE

\title{
Superconductivity emerging from a stripe charge order in $\mathrm{IrTe}_{2}$ nanoflakes
}

\author{
Sungyu Park ${ }^{1,14}$, So Young Kim²,2,14, Hyoung Kug Kim², Min Jeong Kim¹,3, Taeho Kim,3, Hoon Kim ${ }^{1,2}$, \\ Gyu Seung Choi ${ }^{1,2}$, C. J. Won (10 ${ }^{4,5}$, Sooran Kim (1) ${ }^{6}$, Kyoo Kim (10 ${ }^{7}$, Evgeny F. Talantsev (10 ${ }^{8,9}$, \\ Kenji Watanabe (iD ${ }^{10}$, Takashi Taniguchi (1) ${ }^{11}$, Sang-Wook Cheong (1) 4,5,12, B. J. Kim (1) 1,2, H. W. Yeom (1) 1,2, \\ Jonghwan Kim ${ }^{1,2,3 凶}$, Tae-Hwan Kim (i) ${ }^{2,5,13 凶} \&$ Jun Sung Kim (i) ${ }^{1,2 凶}$
}

Superconductivity in the vicinity of a competing electronic order often manifests itself with a superconducting dome, centered at a presumed quantum critical point in the phase diagram. This common feature, found in many unconventional superconductors, has supported a prevalent scenario in which fluctuations or partial melting of a parent order are essential for inducing or enhancing superconductivity. Here we present a contrary example, found in $\operatorname{IrTe} e_{2}$ nanoflakes of which the superconducting dome is identified well inside the parent stripe charge ordering phase in the thickness-dependent phase diagram. The coexisting stripe charge order in $\mathrm{IrTe}_{2}$ nanoflakes significantly increases the out-of-plane coherence length and the coupling strength of superconductivity, in contrast to the doped bulk $\mathrm{IrTe}_{2}$. These findings clarify that the inherent instabilities of the parent stripe phase are sufficient to induce superconductivity in $\mathrm{IrTe}_{2}$ without its complete or partial melting. Our study highlights the thickness control as an effective means to unveil intrinsic phase diagrams of correlated van der Waals materials.

\footnotetext{
${ }^{1}$ Center for Artificial Low Dimensional Electronic Systems, Institute for Basic Science, Pohang, Korea. ${ }^{2}$ Department of Physics, Pohang University of Science and Technology, Pohang, Korea. ${ }^{3}$ Department of Materials Science and Engineering, Pohang University of Science and Technology, Pohang, Korea. ${ }^{4}$ Laboratory for Pohang Emergent Materials, Pohang Accelerator Laboratory, Pohang, Korea. ${ }^{5}$ Max Planck POSTECH/Korea Research Initiative, Pohang, Korea. ${ }^{6}$ Department of Physics Education, Kyungpook National University, Daegu, Korea. ${ }^{7}$ Korea Atomic Energy Research Institute (KAERI), Yuseong-gu, Daejeon, Korea. ${ }^{8}$ M.N. Mikheev Institute of Metal Physics, Ural Branch, Russian Academy of Sciences, Ekaterinburg, Russia. ${ }^{9}$ NANOTECH Centre, Ural Federal University, Ekaterinburg, Russia. ${ }^{10}$ Research Center for Functional Materials, National Institute for Materials Science, Tsukuba, Japan. ${ }^{11}$ International Center for Materials Nanoarchitectonics, National Institute for Materials Science, Tsukuba, Japan. ${ }^{12}$ Rutgers Center for Emergent Materials and Department of Physics and Astronomy, Rutgers University, Piscataway, NJ, USA. ${ }^{13}$ Asia Pacific Center for Theoretical Physics (APCTP), Pohang, Korea.

${ }^{14}$ These authors contributed equally: Sungyu Park, So Young Kim. ${ }^{凶}$ email: jonghwankim@postech.ac.kr; taehwan@postech.ac.kr; js.kim@postech.ac.kr
} 
T ransition metal dichalcogenides (TMDCs) provide a prototypical quasi-two-dimensional system, possessing various electronic instabilities to periodic charge modulations ${ }^{1}$. These instabilities often induce complex charge-density-wave (CDW) phases with different commensurability conditions ${ }^{2-5}$, sometimes associated with Mott- ${ }^{2}$ or excitonic insulating ${ }^{6}$ phases. Upon chemical doping ${ }^{4,5,7,8}$, these phases are commonly driven into a superconducting phase, resulting in a characteristic domeshaped phase diagram, reminiscent of those found in other unconventional superconductors ${ }^{9-11}$ (Fig. 1a). Understanding the complex interplay between charge ordering and superconducting instabilities in TMDCs is a long standing issue, which has often been hampered by presence of quenched disorders, introduced in chemical doping. Recently, utilising the weak van der Waals ( $\mathrm{vdW}$ ) coupling between layers, TMDCs were found to be thinned down to atomic length scale ${ }^{12}$, comparable with the coherence lengths of their various electronic orders. This offers another effective way to tune stability or properties of the competing phases, as demonstrated for $1 T-\mathrm{TaS}_{2}{ }^{13-15}$ and $\mathrm{NbSe}_{2}{ }^{16,17}$, in which distinct thickness dependence of the transition temperatures is observed for superconducting and CDW phases.

$\mathrm{IrTe}_{2}$ is one of the TMDCs in vdW structure (Fig. 1b), showing a similar dome-shaped superconducting phase diagram ${ }^{18-23}$. $\mathrm{IrTe}_{2}$ undergoes a stripe charge ordering transition at $T_{s} \sim 260 \mathrm{~K}$, and by suppressing it with e.g. chemical doping ${ }^{18-23}$ the superconducting phase eventually appears, similar to TMDCs hosting the parent $\mathrm{CDW}$ orders ${ }^{4,5,7,8}$. The stripe order in $\mathrm{IrTe}_{2}$, however, is accompanied by the first-order structural transition involving in-plane Ir-Ir dimerization and interlayer $\mathrm{Te}-\mathrm{Te}$ depolymerisation ${ }^{24,25}$, which forms stripe patterns with a predominant period of $5 a_{0}\left(a_{0}\right.$, the $a$ axis lattice constant $)$ as depicted in Fig. $1 c$ and $d^{19,26}$. No clear evidence of gap opening in the Fermi surface (FS) is observed ${ }^{27,28}$, unlike the typical CDW gap formation in $\mathrm{TMDCs}^{29}$. Instead, FS reconstruction to the socalled cross-layer two-dimensional (2D) state ${ }^{30,31}$ occurs due to suppression of the density of states (DOS) in the planes of Ir-Ir dimers running across the vdW gaps. These aspects suggest that the relationship between the stripe and superconducting orders in $\mathrm{IrTe}_{2}$ may differ significantly from those of other TMDCs, as indicated by recent discoveries on the superconductivity in quenched or thinned $\mathrm{IrTe}_{2}$ crystals $^{32-34}$. Here using Raman spectroscopy, scanning tunnelling microscopy, and transport property measurements, we found that the parent stripe phase encompasses the whole superconducting dome in the thicknessdependent phase diagram (Fig. 1a). This unusual coexistence of the stripe and superconducting orders significantly increases the interlayer coherence length and the coupling strength of superconductivity in $\mathrm{IrTe}_{2}$ nanoflakes, revealing the collaborative role of the stripe order to the superconductivity in $\mathrm{IrTe}_{2}$.

\section{Results}

Thickness dependent phase transitions. In order to vary the thickness of IrTe 2 , we employed the mechanical exfoliation method of single crystals and obtained thin flakes with thickness $(d)$ down to $\sim 10 \mathrm{~nm}$, which show a systematic thickness dependence of the in-plane resistivity $\rho$ (Fig. 1e). For the temperature sweeps in both directions, we took a slow cooling rate of $\sim 0.5 \mathrm{~K} / \mathrm{min}$, in order to minimise inhomogeneous domain formation of the stripe-chargeordered and charge-disordered phases, found in rapid-cooled $\mathrm{IrTe}_{2}$ crystal $^{32,33,35}$. The temperature dependent $\rho(T)$ follows a metallic temperature dependence with abrupt changes at $T_{s, \mathrm{dn}}$ and $T_{s, \mathrm{up}}$, due to the first-order stripe ordering transition as found in bulk crystal $^{24,25}$. These resistive anomalies across the transitions, however, become much smaller in size with reducing $d$, and eventually disappears for $d<50 \mathrm{~nm}$. This does not mean full suppression of a
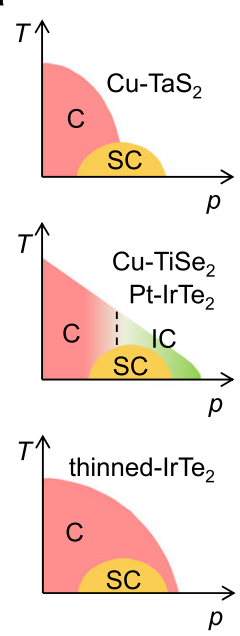

b
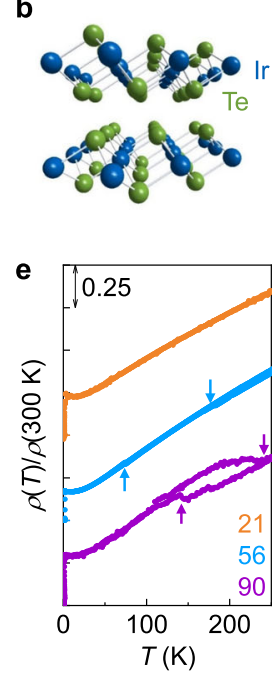

c

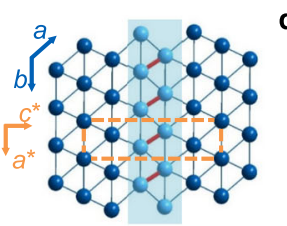

d

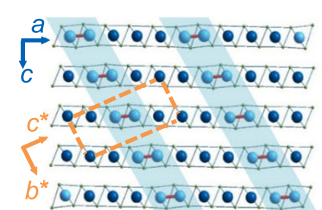

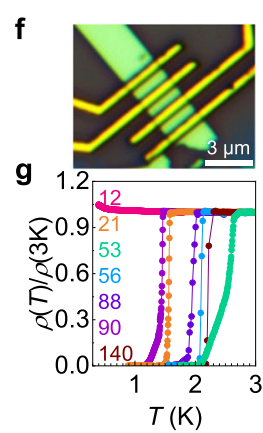

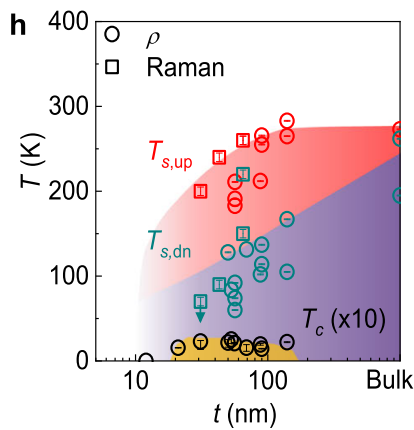

Fig. 1 Structure and phase diagram of IrTe $\mathbf{2}_{\mathbf{2}}$ nanoflakes. a Schematic phase diagrams of TMDCs as a function of control parameter $p$, showing commensurate (C), incommensurate charge order (IC), and superconductivity (SC). Three different types of dome-shaped superconducting phase diagram, where the dome lies at the centre of a presumed quantum critical point (top), near the C-IC transition line (middle), or well inside the parent order (bottom). $\mathbf{b}$ Crystal structure of $\operatorname{IrTe} e_{2}$. $\mathbf{c}$, d Schematic illustrations of the stripe order in $\operatorname{IrTe} e_{2}$ below $T_{s}$. The Ir-Ir dimerization (red) with a modulation vector $\mathbf{q}=\left(\frac{1}{5}, 0, \frac{1}{5}\right)$ is depicted (blue shade) on the triangular Ir layer (c) and across the stacked layers (d). The crystallographic axes for the high- $T(a, b$, and $c)$ and the low- $T\left(a^{\star}, b^{\star}\right.$, and $\left.c^{\star}\right)$ structures are shown, together with the unit cell of the stripe phase (orange box). e Temperature dependence of the normalised resistivity $\rho(T) / \rho(300 \mathrm{~K})$ for $\operatorname{IrTe} e_{2}$ crystals with thickness $(d)$ of 21,56 , and $90 \mathrm{~nm}$. For clarity, $\rho(T) / \rho(300 \mathrm{~K})$ curves are offset vertically. Transition temperatures $T_{\text {s,up }}$ and $T_{s, \text { dn }}$ are determined (arrows) in opposite temperature sweeps. f Optical microscope image of a 56-nm-thick IrTe $\mathrm{crystal}$. $\mathbf{g} \rho(T) / \rho$ $(3 \mathrm{~K})$ curves for $\operatorname{IrTe} e_{2}$ crystals with $d=12-140 \mathrm{~nm}$. h Phase diagram of IrTe 2 nanoflakes as a function of thickness $d$, obtained by transport (circle) and Raman spectroscopy (square) measurements. The transition temperatures $T_{s, u p}$ (red) and $T_{s, d n}$ (blue) during warming and cooling are plotted with the superconducting transition temperature $T_{c}$ (black), scaled by a factor of 10 for clarity. The error bars from the resistivity and Raman spectroscopy are defined by the width of the corresponding resistive transitions and the temperature step of $5 \mathrm{~K}$ between the measurements, respectively. 

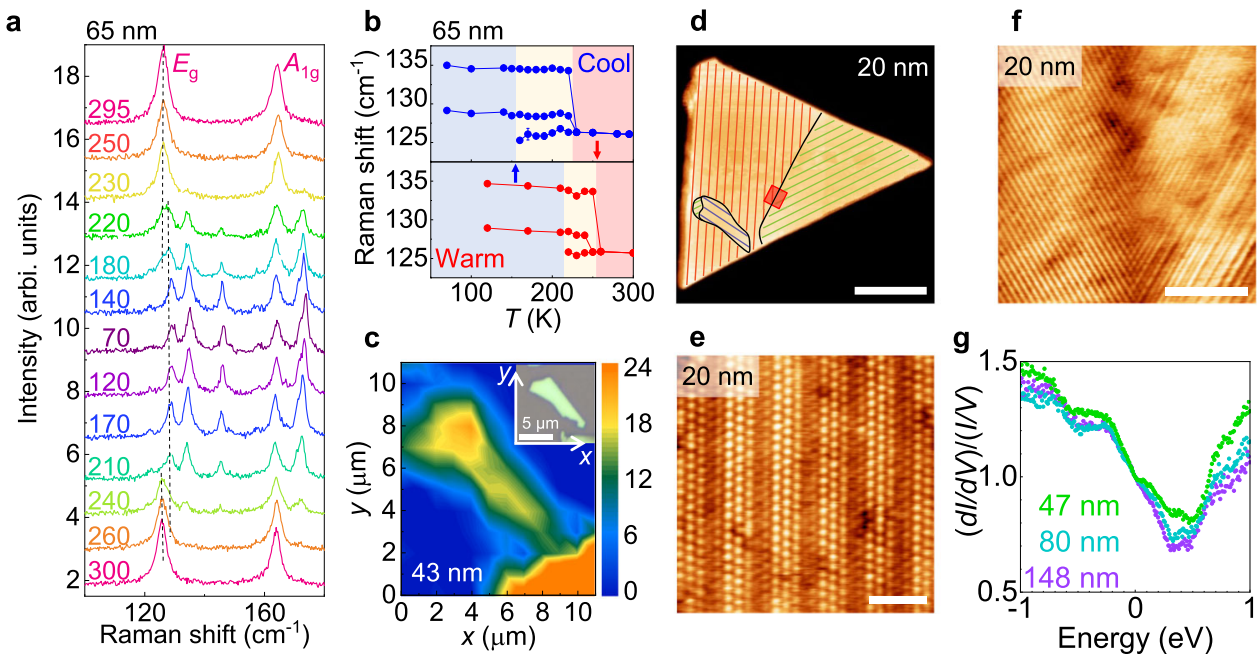

Fig. 2 Stripe charge ordering formation in IrTe $\mathbf{2}$ nanoflakes. $\mathbf{a}, \mathbf{b}$ Raman spectra (a) and corresponding temperature dependent Raman frequency (b) of a 65-nm-thick IrTe $\mathrm{T}_{2}$ nanoflake at various temperatures during cool-down and warm-up procedures. At $T<T_{s}$, the Raman modes, $E_{g}$ at $126 \mathrm{~cm}^{-1}$ and $A_{1 g}$ at $165 \mathrm{~cm}^{-1}$, split into multiple peaks as the flake forms the stripe charge order in a. The temperature ranges for the normal (red), the stripe (blue), and intermediate coexistence (yellow) phases, are identified in $\mathbf{b}$, during cooling (upper panel) and warming (lower panel). Transition temperatures $T_{s, \text { dn }}$ and $T_{s, \text { up }}$ are indicated by the arrows, respectively. c Spatial profile of Raman intensity map for $129 \mathrm{~cm}^{-1} \mathrm{for}_{\text {a }} 43-\mathrm{nm}$-thick IrTe $\mathrm{e}_{2}$ nanoflake at $T \sim 70 \mathrm{~K}$. Inset:

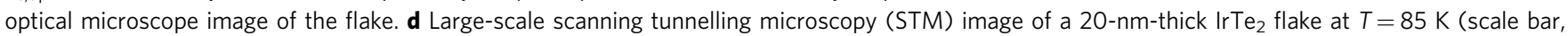
$300 \mathrm{~nm}$ ). The flake has only stripe-phase charge-ordered domains, illustrated by red, green, and blue lines. Black lines indicate domain boundaries between three equivalent stripe-phase charge-ordered domains. e Atomically resolved STM image of $\mathbf{d}$ at $T=85 \mathrm{~K}$ representing a uniform striped area with $5 \times$ 1 surface reconstruction (scale bar, $2 \mathrm{~nm}$ ). $\mathbf{f}$ Zoomed-in STM image of two charge-ordered phases indicated by red square in $\mathbf{d}$ showing that the two phases coexist at the boundary (scale bar, $20 \mathrm{~nm}$ ). $\mathbf{g}$ Scanning tunnelling spectroscopy (STS) spectra at $T=85 \mathrm{~K}$ taken on IrTe $\mathrm{n}_{2}$ nanoflakes with $d=47,80$, and $148 \mathrm{~nm}$, as indicated in the plot.

the stripe order in thin nanoflakes, since the size of the resistive anomaly is known to be strongly suppressed by introducing strain, reducing the sample volume, and increasing the cooling rate even in bulk samples ${ }^{34}$. Rather, the stripe order is found to be stable in all the nanoflakes we studied, as discussed below (Fig. 2). At low temperatures, all the nanoflakes, except the thinnest one with $d=$ $12 \mathrm{~nm}$, exhibit a superconducting transition as found in the temperature-dependent normalised resistance $\rho(T) / \rho(3 \mathrm{~K})$ (Fig. 1g). The superconducting transition temperature, defined as a $50 \%$ resistive transition, is $T_{c}=1.43-2.64 \mathrm{~K}$, somewhat lower than $T_{c} \sim 3$ $\mathrm{K}$ for the optimally doped bulk $\mathrm{IrTe}_{2}{ }^{18,19}$, mostly due to the $2 \mathrm{D}$ nature as discussed below (Fig. 3). Unlike the stripe charge ordering transition, the superconductivity is found to be quite stable in nanoflakes. The superconducting transition temperatures and widths remain almost the same in different thermal cycling (Supplementary Fig. 7).

The stripe ordering transition of $\mathrm{IrTe}_{2}$ nanoflakes is characterised by Raman spectroscopy. For bulk $\operatorname{IrTe}_{2}$, two Raman active modes, $E_{\mathrm{g}}$ at $126 \mathrm{~cm}^{-1}$ and $A_{1 \mathrm{~g}}$ at $165 \mathrm{~cm}^{-1}$, in a trigonal structure (space group $P \overline{3} m 1$ ) split into multiple peaks due to the lowered symmetry and the emergence of a super unit cell for the stripe order below $T_{s}^{30,36}$. Raman spectra taken from the $65-\mathrm{nm}$ thick nanoflake with sequential decrease and increase of temperature (Fig. 2a) reveal that both $E_{\mathrm{g}}$ and $A_{1 \mathrm{~g}}$ Raman modes at room temperature split into multiple Raman modes at $T=70$ $\mathrm{K}$, well below $T_{s}$, consistent with previous studies on the bulk ${ }^{36}$. This behaviour is also shown for the flakes with different thicknesses (Supplementary Fig. 2a and Fig. 3a) and confirms formation of the stripe charge order in our $\mathrm{IrTe}_{2}$ nanoflakes.

In $\mathrm{IrTe}_{2}$ nanoflakes, however, the temperature dependence of the stripe phase evolution is distinct from the bulk case. We found that below $T_{s}$ there is an intermediate temperature range (yellow range in Fig. 2b) where the high temperature Raman modes coexist with the low temperature modes, for both temperature sweeps. This contrasts to the abrupt change found in bulk $\mathrm{IrTe}_{2}$ with a negligible coexistence range ${ }^{36}$ and indicates macroscopic phase separation of the normal and stripe phases in $\mathrm{IrTe}_{2}$ nanoflakes at the intermediate temperature. We defined $T_{s, \mathrm{dn}}$ and $T_{s, \text { up }}$ as the temperatures where the contribution from the normal and stripe phase disappears during the cool-down and warm-up procedures, respectively (Fig. 2b). As the flake thickness decreases, the transition temperatures monotonically decrease, which are in good agreement with those from $\rho(T)$ (Fig. 1h). In addition, we measured the Raman spectroscopy on $\operatorname{IrTe}_{2}$ flakes, cooled down to $T=4 \mathrm{~K}$, just above $T_{c} \sim 2 \mathrm{~K}$. For $\operatorname{IrTe}_{2}$ flakes with various thicknesses from $10 \mathrm{~nm}$ to $174 \mathrm{~nm}$, which cover the whole superconducting dome in the thickness-dependent phase diagram, we observed clear splitting of Raman modes, consistent with the stripe-charge-order formation (Supplementary Fig. 3). These results clearly show that the region of the stripe phase overlaps with the entire superconducting dome in a wide range of thickness.

Coexistence of stripe order and superconductivity. This phase diagram significantly differs from the doping-dependent phase diagram of bulk $\operatorname{IrTe}_{2}{ }^{18-23}$. In the bulk case, the stripe and superconducting phases are mutually exclusive, and the coexisting region appears in the very narrow doping range ${ }^{18-23}$. Even in this coexisting phase, two ordered phases are macroscopically separated $^{22,23}$. Such a macroscopic phase separation is also suggested in the super-cooled case $32,33,35$. However, in $\mathrm{IrTe}_{2}$ nanoflakes, the stripe phase completely covers the whole regions of the sample, as confirmed by the spatial mapping of Raman signal, taken at $\sim 70 \mathrm{~K}$ well below $T_{s}$ (Fig. $2 \mathrm{c}$ ). The intensity map of the $129 \mathrm{~cm}^{-1}$ Raman mode, which is the hallmark of the stripe phase, reveals a strong Raman intensity profile over the entire nanoflake as in the optical microscope image (Fig. $2 \mathrm{c}$ inset), while the signal from the normal phase is absent (Supplementary Fig. 2c). This indicates that the stripe phase dominantly prevails in the 

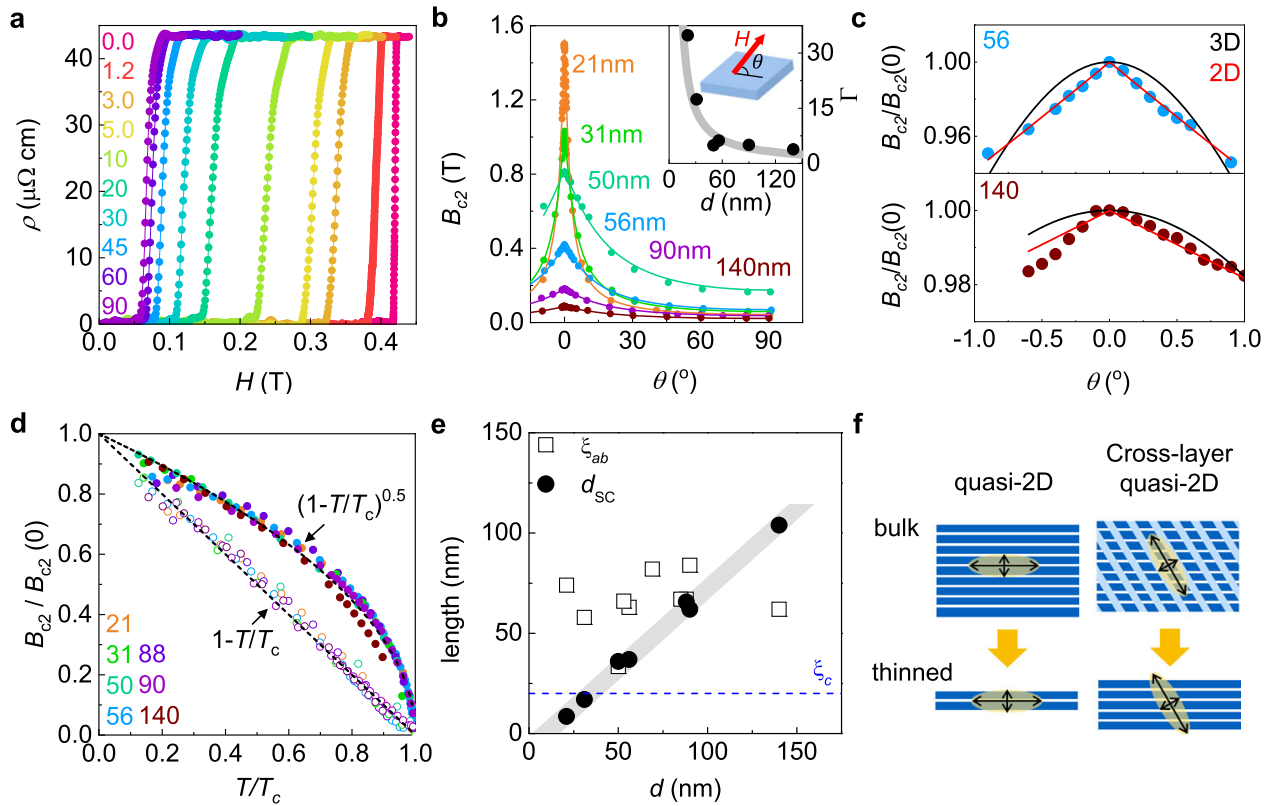

Fig. 3 Two dimensional superconductivity of $\mathbf{I r T e} \mathbf{2}_{2}$ nanoflakes. a, Magnetic field dependence of $\rho(H)$ of a 56-nm-thick IrTe 2 nanoflake, measured with different field orientations $\theta$ at $T=0.35 \mathrm{~K}$. b. Upper critical field $B_{c 2}$ as a function of field angle $\theta$ for $\operatorname{IrTe} e_{2}$ nanoflakes with different thickness ( $d$ ) at $T=0.35$ $\mathrm{K}$, together with the fit (solid line) to the 2D Tinkham model. Inset: the anisotropy factor $\Gamma=B_{c 2}^{a b} / B_{c 2}^{c}$ as a function of $d$, following $1 / d$ dependence (grey line). Schematic illustration shows the field orientation $\theta$. c, Angle dependence of $B_{c 2}(\theta)$ of $\operatorname{IrTe} e_{2}$ nanoflakes with $d=56$ and $140 \mathrm{~nm}$ at $T=0.35 \mathrm{~K}$. Good agreement with the 2D Tinkham model (red), rather than the 3D Ginzburg-Landau model (black), confirms the 2D superconductivity. d, Normalised $B_{c 2} /$ $B_{c 2}(0)$ as a function of $T / T_{c}$ for $\operatorname{Ir} T e_{2}$ nanoflakes with different $d$. All data collapse into dashed lines described by $1-T / T_{c}$ and $\left(1-T / T_{c}\right)^{1 / 2}$ for $B \| c$ (open circles) and $B \| a b$ (solid circles), respectively. e, Ginzburg-Landau coherence length $\xi_{a b}$ (square) and the effective superconducting thickness $d_{\mathrm{SC}}$ (circle) as a function of $d . \xi_{a b}$ is nearly independent of $d$, whereas $d_{\mathrm{SC}}$ grows linearly with $d\left(d_{\mathrm{SC}} \sim 0.8 d\right)$ and exceeds $\xi_{c}$ of doped bulk IrTe $\mathrm{e}_{2}$. f, Schematic illustration of the size effect of vdW superconductors. In normal vdW superconductors with a large anisotropy $\xi_{c} \ll \xi_{a b}, 2 \mathrm{D}$ superconductivity appears only for a-few-layerthick crystals. In $\operatorname{IrTe} e_{2}$ with a stripe order and the resulting cross-layer quasi-2D state, the increased $\xi_{c} \sim \xi_{a b}$ induces $2 \mathrm{D}$ superconductivity in relatively thick nanoflakes.

macroscopic length scale and serves as a normal state for the superconductivity, in contrast to the doped bulk $\mathrm{IrTe}_{2}$ case.

The dominant stripe phase formation is further confirmed by scanning tunnelling microscopy (STM) for a representative nanoflake with $d=20 \mathrm{~nm}$ (Fig. $2 \mathrm{~d}$ ). At room temperature, the hexagonal lattice of top-most Te atoms is clearly resolved by STM in all $\mathrm{IrTe}_{2}$ nanoflakes (Supplementary Fig. 4b). When cooled down below the stripe ordering temperature $\left(T_{\mathrm{STM}}=85 \mathrm{~K}<T_{s}\right)$, the ultrathin nanoflake develops clear stripe patterns with a period of $5 a_{0}$ due to the charge ordering and dimerization of Ir atoms (Fig. 2e), as observed in bulk crystals ${ }^{25,26}$. By scanning over the nanoflake with sufficient spatial resolution (Fig. 2f), we confirm that the whole surface of the flake hosts one or two predominant stripe phases among three energetically equivalent phases (Fig. 2d), and the stripe patterns are often oriented nearly parallel to the long edges of nanoflakes. In thicker nanoflakes (Supplementary Fig. 5a), stripe domain patterns became more complex, similar to bulk $\operatorname{IrTe}_{2}{ }^{35}$. We note that even with fast cooling at $\sim 1 \mathrm{~K} / \mathrm{sec}$, neither thin nor thick nanoflakes show the hexagonal phase that is often observed in either super-cooled or doped bulk $\operatorname{IrTe}_{2}{ }^{22,35}$ and considered to be responsible for the superconductivity. Consistently, in the scanning tunnelling spectroscopy (STS) measurements on $\mathrm{IrTe}_{2}$ nanoflakes, we observe similar local DOS over the wide range of thicknesses. In STS spectra (Fig. 2g), obtained on different $\mathrm{IrTe}_{2}$ nanoflakes with $47 \leq d \leq 148 \mathrm{~nm}$, the local spectral features are qualitatively consistent with the total DOS for the stripe phase of bulk $\operatorname{IrTe}_{2}$, as estimated using first principle calculations ${ }^{30}$. Our results from STM and Raman spectroscopy provide strong evidence that the superconductivity emerges from the preexisting stripe phase in $\mathrm{IrTe}_{2}$ nanoflakes.

2D superconductivity. Now we focus on the effect of the underlying stripe order on the superconducting properties. To address this issue, we investigated the upper critical field $B_{c 2}$ of each nanoflake as a function of field orientation below $T_{c}$. Figure $3 \mathrm{a}$ shows the resistivity $\rho(H)$ curves of a representative nanoflake with $d=56 \mathrm{~nm}$, collected at $T=0.35 \mathrm{~K}$ under a magnetic field, for which the angle $\theta$ is defined with respect to the $a b$ plane. The anisotropy of $B_{c 2}, \Gamma=B_{c 2}^{a b} / B_{c 2}^{c} \sim 5.3$ for $d=56 \mathrm{~nm}$, becomes stronger with lowering $d$ and reaches up to $\Gamma \sim 38$ for $d=21 \mathrm{~nm}$ (Fig. 3b), an order of magnitude larger than $\Gamma \sim 2$ of doped bulk $\mathrm{IrTe}_{2}{ }^{37}$. This large increase in $\Gamma$ with moderate changes in $T_{c}$ (Fig. $1 \mathrm{~g}$ ) and the in-plane coherence length $\xi_{a b}(0)$ (Fig. 3e) can only be explained by $2 \mathrm{D}$ superconductivity. In the Tinkham model of $2 \mathrm{D}$ superconductivity ${ }^{38}$, the angle dependent $B_{c 2}(\theta)$ at the zero-temperature limit is described by $\left|B_{c 2}(\theta) \sin \theta / B_{c 2}^{c}\right|+$ $\left(B_{c 2}(\theta) \cos \theta / B_{c 2}^{a b}\right)^{2}=1$, where $B_{c 2}^{a b}=\left(\sqrt{12} \Phi_{0}\right) /\left(2 \pi \xi_{a b}(0) d_{\mathrm{SC}}\right)$ and $B_{c 2}^{c}=\Phi_{0} /\left(2 \pi \xi_{a b}(0)^{2}\right)\left(\Phi_{0}\right.$, a flux quantum). Thus by reducing the effective thickness of the superconducting layer $d_{\mathrm{SC}}, \Gamma$ becomes large with a constant $\xi_{a b}(0)$, and a discontinuous cusp in the $B_{c 2}(\theta)$ curve near $\theta=0^{\circ}$ is expected. These predictions are distinct from those of the Ginzburg-Landau model for anisotropic threedimensional (3D) superconductors ${ }^{38}$, as described by $B_{c 2}(\theta)=$ $B_{c 2}\left(0^{\circ}\right) / \sqrt{\Gamma^{2} \sin ^{2} \theta+\cos ^{2} \theta}$. All $B_{c 2}(\theta)$ curves exhibit a clear cusp near $\theta=0^{\circ}$ and are successfully fitted by the 2D Tinkham model rather than the anisotropic 3D model (Fig. 3b and Supplementary 
Fig. 9). For $d=140 \mathrm{~nm}$, the thickest sample, $B_{c 2}(\theta)$ slightly deviates from the $2 \mathrm{D}$ model, but still far from $3 \mathrm{D}$ model (Fig. $3 \mathrm{c}$ ). These results demonstrate that $\operatorname{IrTe}_{2}$ nanoflakes with $d \leq 140 \mathrm{~nm}$ clearly show the $2 \mathrm{D}$ superconductivity.

The temperature dependence of $B_{c 2}(T)$ under in-plane $(B \| a b)$ and out-of-plane $(B \| c)$ magnetic fields further confirms the $2 \mathrm{D}$ superconductivity of $\operatorname{IrTe}_{2}$ nanoflakes. For seven samples with different $d$ 's, we determined $B_{c 2}(T)$ by taking $50 \%$ of the resistive transition as a function of the normalised temperature $t=T / T_{c}$ (Fig. 3d and Supplementary Fig. 8). The out-of-plane $B_{c 2}^{c}(t)$ is almost the same, following the linear dependence (Fig. $3 \mathrm{~d}$ ), as observed in the doped bulk sample ${ }^{37}$. The in-plane $B_{c 2}^{a b}(t)$ increases strongly with lowering $d$, but the normalised $B_{c 2}(t) /$ $B_{c 2}(0)$ curves for all samples collapse into a single curve following the 2D Ginzburg-Landau model ${ }^{38}, B_{c 2}^{a b}(t)=\frac{\Phi_{0}}{2 \pi} \frac{\sqrt{12}}{\xi_{a b} d_{\mathrm{SC}}}(1-t)^{1 / 2}$. Using $\xi_{a b}(0)$, estimated from the observed $B_{c 2}^{c}(0)$, we obtained $d_{\mathrm{SC}}$ is $\sim 80 \%$ of the measured thickness $d$ (Fig. 3e). Considering that $2 \mathrm{D}$ superconductivity is induced at $d\left(\sim d_{\mathrm{SC}}\right)$ smaller than the out-of-plane coherence length $\xi_{c}$, i.e. $d<\xi_{c}$, we conclude that $\xi_{c}(0)$ of $\mathrm{IrTe}_{2}$ nanoflakes should be larger than the maximum value of $d_{\mathrm{SC}} \sim 100 \mathrm{~nm}$, obtained in experiments (Fig. 3e), This value is significantly larger than the typical $\xi_{c}(0) \sim 25 \mathrm{~nm}$ of doped $\operatorname{IrTe}_{2}$ bulk samples ${ }^{37}$, and comparable to the in-plane coherence length $\xi_{a b}(0) \sim 70 \mathrm{~nm}^{39}$ (Fig. 3e). These findings clearly indicate that the superconducting characteristics of $\mathrm{IrTe}_{2}$ nanoflakes are distinct from those of the doped $\mathrm{IrTe}_{2}$.

Characteristics of superconductivity coexisting stripe order. The drastically increased $\xi_{c}(0)$ in $\mathrm{IrTe}_{2}$ nanoflakes is a consequence of the coexisting stripe order. In anisotropic superconductors, the interlayer coherence length $\xi_{c}(0)$ is determined by the superconducting gap $\left(\Delta_{\mathrm{SC}}\right)$ and the Fermi velocity $\left(v_{\mathrm{F}}^{c}\right)$, i.e. $\xi_{c}(0) \propto v_{\mathrm{F}}^{c} /$ $\Delta_{\mathrm{SC}}$. Assuming a similar $\Delta_{\mathrm{SC}}, \xi_{c}(0) / \xi_{a b}(0) \approx v_{\mathrm{F}}^{c} / v_{\mathrm{F}}^{a b} \gtrsim 1$ seems incompatible with the vdW structure of $\operatorname{IrTe}_{2}$. This however can be explained by considering the coexisting stripe order. In the stripe phase of $\mathrm{IrTe}_{2}$, Ir-Ir dimerization and Te-Te depolymerisation produce conducting planes between the dimer planes, running across the vdW gaps (Fig. 1d). This cross-layer 2D conducting state $^{30}$ affects the electronic structure such that the interlayer $v_{\mathrm{F}}^{c}$ is even larger than the in-plane $v_{\mathrm{F}}^{a b}$, which greatly increases $\xi_{c}(0)$ (Fig. 3f). Unlike the conventional vdW superconductors in which 2D superconductivity can only be induced in a-few-layer-thick crystals, $\mathrm{IrTe}_{2}$ hosts $2 \mathrm{D}$ superconductivity in the relatively thick crystals due to the microscopically coexisting stripe order.

The distinct superconducting nature in $\mathrm{IrTe}_{2}$ nanoflakes is also found in their superconducting gap as compared to the doped bulk. Figure $4 \mathrm{a}$ presents the current-voltage (IV) characteristics at different temperatures for a representative nanoflake with $d=21 \mathrm{~nm}$. Near $T_{c} \approx T_{\mathrm{BKT}}$, they follow Berezinskii-Kosterlitz-Thouless transition for $2 \mathrm{D}$ superconductivity (Fig. 4b), in which the exponent $\alpha$ extracted from $V \propto I^{\alpha}$ crosses $\alpha=3$ at $T_{\text {BKT. }}$. Well below $T_{c}$, the self-field critical current density $J_{c, s f}(T)$ can be obtained from the IV characteristics with variation of temperature, which is proportional to temperature dependent London penetration depth $\lambda(T)$ for $d \ll \lambda^{40,41}$. From the critical current $I_{c}(T)$, at which the measured voltage jumps due to the superconducting-to-normal transition, we obtained the corresponding $J_{c, s f}(T)$ and $\lambda(T)$ curves (Fig. $4 c$ and Supplementary Fig. 10), which can be nicely reproduced by the fit based on BCS theory using $\Delta_{\mathrm{SC}} \approx 0.38 \mathrm{meV}$. The superconducting gap $\left(\Delta_{\mathrm{SC}}\right)$, taken from $\mathrm{IrTe}_{2}$ nanoflakes with different thicknesses, varies linearly with their $T_{c}$ with a superconducting gap ratio of $2 \Delta_{\mathrm{SC}} / k_{\mathrm{B}} T_{c} \sim 5.3$, which is much larger than the BCS value of 3.53 and $2 \Delta_{\mathrm{SC}} / k_{\mathrm{B}} T_{c} \sim 3.7$ of doped bulk $\operatorname{IrTe}_{2}{ }^{37,42}$ (Fig. 4d). Thus, superconductivity in $\mathrm{IrTe}_{2}$ nanoflakes is in the strong coupling regime, whereas that of doped bulk $\mathrm{IrTe}_{2}$ is in the weak coupling regime.

\section{Discussion}

Our findings unequivocally emphasise that the superconductivity in $\mathrm{IrTe}_{2}$ nanoflakes, which emerges from the preexisting stripe order, is highly distinct from that in doped bulk $\mathrm{IrTe}_{2}$. On pristine $\mathrm{IrTe}_{2}$ bulk or surface, the $5 a_{0}$ stripe phase undergoes multiple transitions to other nearly-degenerate stripe phases that have different periods such as $8 a_{0}$ and $6 a_{0}$, and also a honeycomb phase $\mathrm{p}^{35,43-45}$. The complex stripe ordering formation is a result of subtle balance between local interactions of Ir-Ir dimerization and Te-Te depolymerisation. These incipient instabilities and the resulting strong electron-lattice coupling of the parent $5 a_{0}$ stripe phase can facilitate pairing interaction for superconductivity in a proper condition and thereby enhancing the superconducting coupling strength as observed in $\mathrm{IrTe}_{2}$ nanoflakes. This coexisting phase of stripe and superconducting orders, however, cannot be accessed by chemical doping, e.g. Pt doping at the Ir sites. A few $\%$ of doping directly perturbs Ir dimerization and melts the stripe order to a quasi-periodic hexagonal order ${ }^{18,22}$. In this case, the onset of superconductivity coincides with disorder-induced melting of the parent order ${ }^{23}$, reminiscent of other TMDCs such as Cu-doped $\mathrm{TiSe}_{2}{ }^{3-5}$.

In contrast, the thickness control of $\mathrm{IrTe}_{2}$ tunes the stripe order without introducing quenched disorders. The stripe order in $\mathrm{IrTe}_{2}$ may be mildly suppressed by the thinning-induced out-ofplane elongation ${ }^{13,46}$ or the substrate-induced in-plane tensile strain as opposite to the pressure effect enhancing $T_{s}^{24}$. Typically, the thinning-induced out-of-plane elongation of $\Delta c / c \sim 0.1 \%$, as found in $\mathrm{TaS}_{2}{ }^{13,46}$ and the substrate-induced in-plane strain of $\Delta a / a \sim 0.1-0.3 \%$ (Supplementary Note 6) are expected in the thinned $\mathrm{IrTe}_{2}$, where $a$ and $c$ are the in-plane and out-of-plane lattice constants, respectively. A recent study on a strained $\mathrm{IrTe}_{2}$ single crystals ${ }^{47}$ revealed that only $\sim 0.1 \%$ of tensile strain induces the transition between the stripe-charge-ordered phases with different periods of $5 a_{0}$ and $6 a_{0}$, which significantly modifies the electronic structures. Our first principle calculations for electronphonon coupling constant $\lambda_{\text {ep }}$ of the $5 a_{0}$ stripe phase show that the in-plane tensile strain is more effective to drive the system to the structural instability and to enhance $\lambda_{\text {ep }}$ than the out-of-plane elongation, which increases $T_{c}$ by a factor of $\sim 3$ (Supplementary Table 1). While the corresponding critical strain is much larger in calculations and also the calculated $T_{c}$ remains lower than the measured $T_{c} \sim 2 \mathrm{~K}$, these observations imply that the stripe phase of $\mathrm{IrTe}_{2}$ is intrinsically in close proximity to the superconducting phase, which can be accessed by reducing thickness or by thermal quenching ${ }^{32,33}$. It has been known that, in the vicinity of full charge order melting, strong electron-phonon coupling ${ }^{48,49}$ can play an important role to promote the superconductivity, as found in the charge-ordered organic metals ${ }^{50,51}$. This appears to be consistent with the enhanced superconducting gap ratio, found in $\mathrm{IrTe}_{2}$ flakes (Fig. 4). Our results unveil the collaborating relationship, rather than the competing one, between the parent stripe and the superconducting orders in $\mathrm{IrTe}_{2}$, highlighting $\operatorname{IrTe}_{2}$ as a unique example among superconducting TMDCs. Further investigations, for examples, scanning tunnelling microscopy and spectroscopy as well as Raman spectroscopy below the $T_{c}$ are highly desirable to identify the local superconducting gap structure along the charge modulation patterns and the electronphonon coupling, which would elucidate the interplay of the stripe-charge-order and superconductivity in $\mathrm{IrTe}_{2}$. 

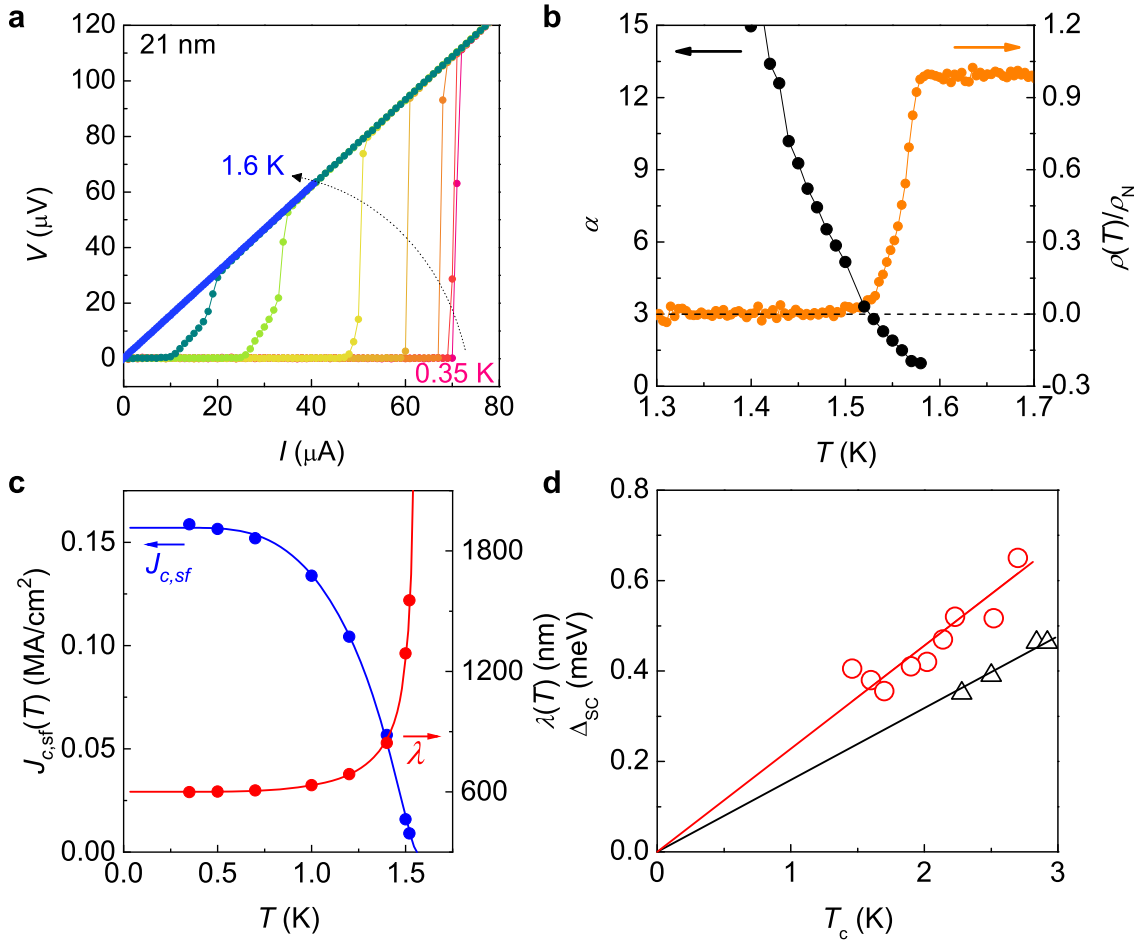

Fig. 4 Strong superconducting coupling in IrTe $\mathbf{T}_{\mathbf{2}}$ nanoflakes. a Current-voltage (IV) characteristics at various temperatures for a representative IrTe 2 nanoflake with $d=21 \mathrm{~nm}$. b Temperature dependence of the normalised resistivity and the exponent $\alpha$ for a 21-nm-thick IrTe 2 nanoflake. Exponent $\alpha$ is determined from the power-law behaviour $V \propto l^{\alpha}$ in the $I V$ curves, as expected by BKT transition. c Critical current density (blue) and the penetration depth (red) as a function of temperature for a 21-nm-thick $\operatorname{IrTe} e_{2}$ nanoflake. Solid lines are the fits to the self-critical-current model described in the text. d Superconducting gap $\Delta_{\mathrm{SC}}$ as a function of $T_{c}$ extracted from the critical current density for $\operatorname{IrTe_{2}}$ nanoflakes with different $d$ (red). The slope of their linear dependence, corresponding to the superconducting gap ratio, $2 \Delta_{\mathrm{SC}} / k_{\mathrm{B}} T_{\mathrm{c}}=5.3$, is much larger than the case of doped bulk $\mathrm{IrTe} \mathrm{e}_{2}$ (black) from refs. ${ }^{37,42}$. This difference confirms the strong coupling nature of the superconductivity in $\operatorname{IrTe}_{2}$ nanoflakes.

\section{Methods}

Single crystal growth and bulk properties. $\operatorname{IrTe}_{2}$ single crystals were synthesised using the Te-flux method ${ }^{31}$. Ir and Te powders were mixed in a stoichiometric ratio Ir:Te $=1: 4$, heated to $1160^{\circ} \mathrm{C}$ for 1 day as sealed in a quartz ampoule, and then cooled. The crystallinity and stoichiometry were confirmed by X-ray diffraction and energy-dispersive X-ray spectroscopy.

Exfoliation and fabrication of nanoflakes. We used mechanical exfoliation of bulk single crystals to obtain thin nanoflakes of $\mathrm{IrTe}_{2}$ on top of a Si/SiO substrate that had been pre-cleaned in acetone, 2-propanol, and deionised water, then treated by oxygen plasma $\left(\mathrm{O}_{2}=10 \mathrm{sccm}, P \sim 100 \mathrm{mTorr}\right)$ for $5 \mathrm{~min}$. All cleaving and handling were done in inert atmosphere $\left(\mathrm{H}_{2} \mathrm{O}<0.1 \mathrm{ppm}, \mathrm{O}_{2}\right.$ $<0.1 \mathrm{ppm}$ ) of pure Ar gas except the atomic force microscopy (AFM) measurements. In some cases, a thin h-BN crystal was subsequently transferred onto the $\mathrm{IrTe}_{2}$ nanoflake in Ar atmosphere. We found that the optical contrast, the AFM thickness, and Raman spectra were unchanged even after 1 week in ambient conditions (Supplementary Fig. 1), indicating that the nanoflakes are stable in ambient conditions. To fabricate devices for electrical measurements, we used conventional e-beam lithography to pattern electrodes on top of $\mathrm{IrTe}_{2}$ nanoflakes with metal deposition of $\operatorname{Cr}(10 \mathrm{~nm}) / \mathrm{Au}(50 \mathrm{~nm})$. The optical microscope image for the typical device is shown in Fig. If.

Transport property measurements. Transport measurements were performed in a cryogenic ${ }^{3} \mathrm{He}$ refrigerator equipped with a superconducting vector magnet $(9 / 2 / 2$ T). Each measurement wire was filtered by a room-temperature $\pi$ filter and lowtemperature $\pi$ and low-pass RC filters at $0.35 \mathrm{~K}$ to minimise the electrical noise on the sample. Electrical resistance was measured in standard four-probe configuration using DC delta mode with bias current $1 \mu \mathrm{A}$ to $10 \mu \mathrm{A}$ determined by the sample resistance and signal to noise ratio. Magnetic fields with desired field orientations were applied by the vector magnet at one cooling without altering sample position.

Raman spectroscopy. Raman spectra were obtained using a confocal microscopy set-up with laser beam size of $\sim 1 \mu \mathrm{m}$ and laser power of $\sim 1 \mathrm{~mW}$. A
HeNe laser $(632.8 \mathrm{~nm})$ was used to excite $\mathrm{IrTe}_{2}$ flakes in an optical cryostat at normal incidence. The Raman signal was collected in the backscattering configuration and analysed using a monochromator equipped with a liquid nitrogen-cooled silicon CCD. Two linear polarizers in the parallel configuration were placed immediately after the laser and before the monochromator to define the polarization of incident and scattered light, respectively. The crystal orientation relative to the polarisation of the incident light was controlled using a half waveplate between a beam splitter and $\operatorname{IrTe}_{2}$ flakes. The sample position was precisely controlled using a piezo stage.

Scanning tunnelling microscopy and spectroscopy. For scanning tunnelling microscopy (STM) and spectroscopy (STS) measurements, $\mathrm{IrTe}_{2}$ nanoflakes were exfoliated in a glove box $\left(\mathrm{H}_{2} \mathrm{O}<0.1 \mathrm{ppm}, \mathrm{O}_{2}<0.1 \mathrm{ppm}\right)$ filled with Ar gas, and transferred onto graphene, grown epitaxially on a $4 \mathrm{H}-\mathrm{SiC}(0001)$ substrate. The samples were then transferred to a ultrahigh vacuum chamber $\left(P \leq 1 \times 10^{-10}\right.$ Torr $)$ for STM/STS measurements without any exposure to air to ensure clean surfaces of $\mathrm{IrTe}_{2}$ nanoflakes. STM images were typically obtained using a bias voltage $V_{\mathrm{b}}=-$ $2.5 \mathrm{~V}$ and a tunnelling current $I_{\mathrm{t}}=20 \mathrm{pA}$ for large-scale imaging; $V_{\mathrm{b}}=15 \mathrm{mV}$ and $I_{\mathrm{t}}=1 \mathrm{nA}$ for charge-ordered stripe phases; $V_{\mathrm{b}}=5 \mathrm{mV}$ and $I_{\mathrm{t}}=2 \mathrm{nA}$ for atomically-resolved images. For STS, we used the lock-in technique with a bias modulation of $7 \mathrm{mV}_{\mathrm{rms}}$.

Density functional theory calculations. Density functional theory (DFT) calculations for electronic structures and the electron-phonon coupling (EPC) were performed by the Quantum Espresso package implementing the pseudo-potential band method and the density functional perturbation theory 52,53. We utilised Perdew-Burke-Ernzerhof sol (PBEsol, revised PBE for solid) ${ }^{54}$ as an exchangecorrelation functional and included the spin-orbit coupling (SOC). The dynamical matrices were calculated using $2 \times 2 \times 2 q$-mesh and $16 \times 10 \times 4 k$-mesh with $40 \mathrm{Ry}$ energy cutoff. We applied the various in-plane tensile strains in the range of $\Delta a / a$ $\sim 2.1-3.1 \%$ and the fixed compressive strain of $\Delta b / b \sim 0.65 \%$ to model the experimental situations (Supplementary Note 6). Atomic positions were optimised in each case. 


\section{Data availability}

The data that support the findings of this study are available from the corresponding authors on request.

Received: 25 October 2020; Accepted: 21 April 2021;

Published online: 26 May 2021

\section{References}

1. Wilson, J. A., Salvo, F. J. D. \& Mahajan, S. Charge-density waves and superlattices in the metallic layered transition metal dichalcogenides. $A d v$. Phys. 24, 117-201 (1975)

2. Sipos, B. et al. From Mott state to superconductivity in $1 T-\mathrm{TaS}_{2}$. Nat. Mater. 7 , 960-965 (2008).

3. Joe, Y. I. et al. Emergence of charge density wave domain walls above the superconducting dome in 1T-TiSe 2 . Nat. Phys. 10, 421-425 (2014).

4. Kogar, A. et al. Observation of a charge density wave incommensuration near the superconducting dome in $\mathrm{Cu}_{x} \mathrm{TiSe}_{2}$. Phys. Rev. Lett. 118, 27002 (2017).

5. Yan, $\mathrm{S}$. et al. Influence of domain walls in the incommensurate charge density wave state of $\mathrm{Cu}$ intercalated 1T-TiSe 2 . Phys. Rev. Lett. 118, 106405 (2017).

6. Cercellier, H. et al. Evidence for an excitonic insulator phase in $1 T-\mathrm{TiSe}_{2}$. Phys. Rev. Lett. 99, 146403 (2007).

7. Wagner, K. E. et al. Tuning the charge density wave and superconductivity in $\mathrm{Cu}_{x} \mathrm{TaS}_{2}$. Phys. Rev. B 78, 104520 (2008).

8. Morosan, E. et al. Superconductivity in $\mathrm{Cu}_{x} \mathrm{TiSe}_{2}$. Nat. Phys. 2, 544-550 (2006).

9. Si, Q. \& Steglich, F. Heavy fermions and quantum phase transitions. Science 329, 1161-1166 (2010)

10. Lee, P. A., Nagaosa, N. \& Wen, X.-G. Doping a Mott insulator: Physics of high-temperature superconductivity. Rev. Mod. Phys. 78, 17 (2006).

11. Stewart, G. Superconductivity in iron compounds. Rev. Mod. Phys. 83, 1589 (2011).

12. Novoselov, K. S. et al. Two-dimensional atomic crystals. Proc. Natl Acad. Sci. USA 102, 10451-10453 (2005).

13. Yoshida, M. et al. Controlling charge-density-wave states in nano-thick crystals of $1 T-\mathrm{TaS}_{2}$. Sci. Rep. 4, 7302 (2014).

14. Yu, Y. et al. Gate-tunable phase transitions in thin flakes of $1 T-\mathrm{TaS}_{2}$. Nat. Nanotechnol. 10, 270-276 (2015).

15. Navarro-Moratalla, E. et al. Enhanced superconductivity in atomically thin $\mathrm{TaS}_{2}$. Nat. Commun. 7, 1-7 (2016).

16. $\mathrm{Xi}, \mathrm{X}$. et al. Strongly enhanced charge-density-wave order in monolayer $\mathrm{NbSe}_{2}$. Nat. Nanotechnol. 10, 765-769 (2015).

17. Xi, X. et al. Ising pairing in superconducting $\mathrm{NbSe}_{2}$ atomic layers. Nat. Phys. 12, 139-143 (2016).

18. Pyon, S., Kudo, K. \& Nohara, M. Superconductivity induced by bond breaking in the triangular lattice of $\operatorname{IrTe}_{2}$. J. Phys. Soc. Jpn. 81, 53701 (2012).

19. Yang, J. J. et al. Charge-orbital density wave and superconductivity in the strong spin-orbit coupled $\mathrm{IrTe}_{2}:$ Pd. Phys. Rev. Lett. 108, 116402 (2012).

20. Kudo, K., Kobayashi, M., Pyon, S. \& Nohara, M. Suppression of structural phase transition in $\mathrm{IrTe}_{2}$ by isovalent Rh doping. J. Phys. Soc. Jpn. 82, 85001 (2013).

21. Kamitani, M. et al. Superconductivity in $\mathrm{Cu}_{x} \mathrm{IrTe}_{2}$ driven by interlayer hybridization. Phys. Rev. B 87, 180501 (2013).

22. Fujisawa, Y. et al. Visualizing the Pt doping effect on surface and electronic structure in $\operatorname{Ir}_{1-x} \mathrm{Pt}_{x} \mathrm{Te}_{2}$ by scanning tunneling microscopy and spectroscopy. J. Phys. Soc. Jpn 84, 43706 (2015).

23. Takubo, $\mathrm{K}$. et al. Commensurate versus incommensurate charge ordering near the superconducting dome in $\mathrm{Ir}_{1-x} \mathrm{Pt}_{x} \mathrm{Te}_{2}$ revealed by resonant $\mathrm{x}$-ray scattering. Phys. Rev. B 97, 205142 (2018).

24. Oh, Y. S., Yang, J. J., Horibe, Y. \& Cheong, S.-W. Anionic depolymerization transition in $\mathrm{IrTe}_{2}$. Phys. Rev. Lett. 110, 127209 (2013).

25. Ko, K.-T. et al. Charge-ordering cascade with spin-orbit Mott dimer states in metallic iridium ditelluride. Nat. Commun. 6, 7342 (2015).

26. Hsu, P.-J. et al. Hysteretic melting transition of a soliton lattice in a commensurate charge modulation. Phys. Rev. Lett. 111, 266401 (2013).

27. Daiki, O. et al. Electronic structure reconstruction by orbital symmetry breaking in $\mathrm{IrTe}_{2}$. J. Phys. Soc. Jpn 82, 93704 (2013).

28. Kim, K. et al. Origin of first-order-type electronic and structural transitions in $\mathrm{IrTe}_{2}$. Phys. Rev. Lett. 114, 136401 (2015).

29. Rahn, D. J. et al. Gaps and kinks in the electronic structure of the superconductor $2 \mathrm{H}-\mathrm{NbSe}_{2}$ from angle-resolved photoemission at $1 \mathrm{~K}$. Phys. Rev. B 85, 224532 (2012).

30. Pascut, G. L. et al. Dimerization-induced cross-layer quasi-two-dimensionality in metallic $\mathrm{IrTe}_{2}$. Phys. Rev. Lett. 112, 86402 (2014).

31. Eom, M. J. et al. Dimerization-induced fermi-surface reconstruction in $\operatorname{IrTe}_{2}$. Phys. Rev. Lett. 113, 266406 (2014).
32. Yoshida, M., Kudo, K., Nohara, M. \& Iwasa, Y. Metastable superconductivity in two-dimensional $\operatorname{IrTe}_{2}$ crystals. Nano Lett. 18, 3113-3117 (2018).

33. Oike, H., Kamitani, M., Tokura, Y. \& Kagawa, F. Kinetic approach to superconductivity hidden behind a competing order. Sci. Adv. 4, eaau3489 (2018).

34. Oike, H. et al. Size effects on supercooling phenomena in strongly correlated electron systems: $\operatorname{IrTe}_{2}$ and $\theta-(\mathrm{BEDT}-\mathrm{TTF})_{2} \mathrm{RbZn}(\mathrm{SCN})_{4}$. Phys. Rev. B 97, 85102 (2018).

35. Kim, H. S. et al. Nanoscale superconducting honeycomb charge order in $\operatorname{IrTe}_{2}$. Nano Lett. 16, 4260-4265 (2016).

36. Glamazda, A., Choi, K.-Y., Lemmens, P., Yang, J. J. \& Cheong, S.-W. Proximity to a commensurate charge modulation in $\operatorname{IrTe}_{2-x} \mathrm{Se}_{x}(x=0$ and 0.45) revealed by Raman spectroscopy. N. J. Phys. 16, 93061 (2014).

37. Yu, D. J. et al. Fully gapped $s$-wave-like superconducting state and electronic structure in $\operatorname{Ir}_{0.95} \mathrm{Pd}_{0.05} \mathrm{Te}_{2}$ single crystals with strong spin-orbital coupling. Phys. Rev. B 89, 100501 (2014).

38. Tinkham, M. Introduction to Superconductivity. (Dover Publications, Inc., New York, 2004)

39. Zhou, S. Y. et al. Nodeless superconductivity in $\mathrm{Ir}_{1-x} \mathrm{Pt}_{x} \mathrm{Te}_{2}$ with strong spin orbital coupling. Europhys. Lett. 104, 27010 (2013).

40. Talantsev, E. F. \& Tallon, J. L. Universal self-field critical current for thin-film superconductors. Nat. Commun. 6, 7820 (2015).

41. Talantsev, E. F. et al. On the origin of critical temperature enhancement in atomically thin superconductors. 2D Mater. 4, 25072 (2017).

42. Wilson, M. N. et al. $\mu \mathrm{SR}$ and magnetometry study of superconducting $5 \% \mathrm{Pt}-$ doped $\mathrm{IrTe}_{2}$. Phys. Rev. B 94, 184504 (2016).

43. Pascut, G. L. et al. Series of alternating states with unpolarized and spinpolarized bands in dimerized $\mathrm{IrTe}_{2}$. Phys. Rev. B 90, 195122 (2014).

44. Rumo, M. et al. Examining the surface phase diagram of $\mathrm{IrTe}_{2}$ with photoemission. Phys. Rev. B 101, 235120 (2020).

45. $\mathrm{Li}, \mathrm{Q}$. et al. Bond competition and phase evolution on the $\mathrm{IrTe}_{2}$ surface. Nat Commun. 5, 5358 (2014).

46. Yoshida, M. et al. Extended polymorphism of two-dimensional material. Nano Lett. 17, 5567-5571 (2017).

47. Nicholson, C. W. et al. Uniaxial strain-induced phase transition in the $2 \mathrm{D}$ topological semimetal $\mathrm{IrTe}_{2}$. Commun. Mater. 2, 1-8 (2021).

48. Merino, J. \& McKenzie, R. H. Superconductivity mediated by charge fluctuations in layered molecular crystals. Phys. Rev. Lett. 87, 237002 (2001).

49. Foussats, A., Greco, A., Bejas, M. \& Muramatsu, A. Cooperative effect of phonons and electronic correlations for superconductivity in cobaltates. Phys. Rev. B 72, 020504 (2005).

50. Kaiser, S. et al. Bandwidth tuning triggers interplay of charge order and superconductivity in two-dimensional organic materials. Phys. Rev. Lett. 105, 206402 (2010).

51. Girlando, A. et al. Charge-order fluctuations and superconductivity in twodimensional organic metals. Phys. Rev. B 89, 174503 (2014).

52. Giannozzi, P. et al. QUANTUM ESPRESSO: a modular and open-source software project for quantum simulations of materials. J. Phys.: Condens. Matter 21, 395502 (2009)

53. Baroni, S., De Gironcoli, S., Dal Corso, A. \& Giannozzi, P. Phonons and related crystal properties from density-functional perturbation theory. Rev Mod. Phys. 73, 515 (2001).

54. Perdew, J. P. et al. Restoring the density-gradient expansion for exchange in solids and surfaces. Phys. Rev. Lett. 100, 136406 (2008).

\section{Acknowledgements}

The authors thank K.T. Ko, G.Y. Jo, Y.K. Bang for fruitful discussion. We also thank H. G. Kim in Pohang Accelerator Laboratory (PAL) for the technical support. This work was supported by the Institute for Basic Science (IBS) through the Center for Artificial Low Dimensional Electronic Systems (no. IBS-R014-D1), the National Research Foundation of Korea (NRF) through SRC (Grant No. NRF-2018R1A5A6075964), and the Max PlanckPOSTECH Center for Complex Phase Materials (Grant No. NRF-2016K1A4A4A01922028). J.K., M.J.K. acknowledge the support from the NRF of Korea grant (Grant No. NRF2017R1C1B2012729 and NRF-2020R1A4A1018935). K.W. and T.T. acknowledge support from the Elemental Strategy Initiative conducted by the MEXT, Japan, Grant Number JPMXP0112101001, JSPS KAKENHI Grant Numbers JP20H00354 and the CREST (JPMJCR15F3), JST. E. F. T. thanks financial support provided by the state assignment of Minobrnauki of Russia (theme Pressure No. AAAA-A18-118020190104-3) and by Act 211 Government of the Russian Federation, contract no. 02.A03.21.0006. S.K. acknowledges the support from the NRF of Korea grant (Grant No. NRF-2019R1F1A1052026) and KISTI supercomputing center (Grant No. KSC-2019-CRE-0172). K.K. acknowledges the support from the NRF of Korea grant (Grant No. 2016R1D1A1B02008461) and Internal R\&D programme at KAERI (Grant No. 5244460-21). SWC was partially supported by the NSF under Grant No. DMR-1629059. 


\section{Author contributions}

S.P., S.Y.K., and J.S.K. conceived the experiments. S.Y.K. and S.P. fabricated the devices. S.P., S.Y.K., G.S.C., and E.F.T. performed transport property measurements and data analysis. H.K.K., H.W.Y., and T.-H.K. performed scanning tunnelling microscopy/ spectroscopy and data analysis. M.J.K., S.Y.K., T. K., H.K., B.J.K., and J.K. performed Raman spectroscopy and data analysis. S.K. and K.K. performed density functional theory calculation and analysis. C.J.W. and S.-W.C. synthesised the bulk crystals. K.W. and T.T. provided boron nitride crystals. S.P., S.Y.K., J.K., T.-H.K., and J.S.K. co-wrote the manuscript. All authors discussed the results and commented on the paper.

\section{Competing interests}

The authors declare no competing interests.

\section{Additional information}

Supplementary information The online version contains supplementary material available at https://doi.org/10.1038/s41467-021-23310-w.

Correspondence and requests for materials should be addressed to J.K., T.-H.K. or J.S.K.

Peer review information Nature Communications thanks the anonymous reviewer(s) for their contribution to the peer review of this work. Peer reviewer reports are available.
Reprints and permission information is available at http://www.nature.com/reprints

Publisher's note Springer Nature remains neutral with regard to jurisdictional claims in published maps and institutional affiliations.

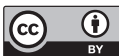

Open Access This article is licensed under a Creative Commons Attribution 4.0 International License, which permits use, sharing, adaptation, distribution and reproduction in any medium or format, as long as you give appropriate credit to the original author(s) and the source, provide a link to the Creative Commons license, and indicate if changes were made. The images or other third party material in this article are included in the article's Creative Commons license, unless indicated otherwise in a credit line to the material. If material is not included in the article's Creative Commons license and your intended use is not permitted by statutory regulation or exceeds the permitted use, you will need to obtain permission directly from the copyright holder. To view a copy of this license, visit http://creativecommons.org/ licenses/by/4.0/.

(C) The Author(s) 2021 